\title{
Técnicas de coaching de bem- estar na mudança do estilo de vida no sistema público de saúde
}

\author{
LUCIANA OQUENDO PEREIRA-LANCHA, ${ }^{\mathrm{I}}$ \\ Daniele KalLas, ${ }^{I I}$ PAULA HELENA DaraN ${ }^{I I I}$ \\ e ANTONIO HERBERT LANCHA JUNIOR ${ }^{\text {IV }}$
}

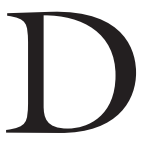
IETAS RESTRITIVAS são o método mais comumente usado para o controle do sobrepeso e obesidade no mundo nos últimos anos. No entanto, artigo publicado na Lancet ( $\mathrm{Ng}$ et al., 2014) mostra que não somente a obesidade está crescendo em todos os países do planeta, mas que também nenhum país do mundo, nesses últimos 33 anos, conseguiu ter sucesso no seu combate. À medida que crescem as taxas globais de doenças crônicas, o foco em estratégias para melhorar os comportamentos de saúde também aumenta, e milhares de diferentes dietas são criadas a cada ano. Os problemas de saúde associados à obesidade são a principal causa de mortalidade nos Estados Unidos, perdendo apenas para problemas associados ao tabagismo. Além disso, o custo das doenças associadas ao sobrepeso para o sistema de saúde é muito elevado.

No Brasil, a situação não é muito diferente: de acordo com o último relatório da Vigilância de Fatores de Risco e Proteção para Doenças Crônicas por Inquérito Telefônico (Vigitel 2017), as Doenças Crônicas Não Transmissíveis (DCNT) foram responsáveis por $68 \%$ de um total de 38 milhões de mortes ocorridas no mundo em 2012, tendo em destaque doenças cardiovasculares $(30,4 \%)$, neoplasias $(16,4 \%)$, doenças respiratórias $(6,0 \%)$ e diabetes $(6,3 \%)$. Estudos mostram que as raízes da doença estão em comportamentos como o tabagismo e a inatividade física, e esta última mata ainda mais do que tabaco, estresse, má alimentação e sono (Mokdad et al., 2004). Portanto, podemos explicar a maioria das doenças com comportamentos simples ligados aos hábitos de estilo de vida, e dessa forma a melhor maneira de combater e prevenir doenças é pela mudança de comportamento.

A necessidade de perder peso é bem compreendida, no entanto o processo é difícil e uma estimativa revela que menos de 1 em 100 pessoas é bem-sucedida em conseguir manter o peso depois da perda. Ironicamente, Fields et al. (2003) mostra, em quase 17 mil crianças com idades entre 9-14 anos, que "fazer" dieta restritiva foi um preditor significativo de ganho de peso. Os autores concluíram que “[...] a longo prazo, fazer dieta para controlar o peso não é eficaz, pode 
realmente promover o ganho de peso". Além disso, Mann et al. (2007), em seu artigo "Medicare's search for effective obesity treatments: Diets are not the answer" [Dietas não são as respostas], avaliaram 31 estudos sobre os resultados de longo prazo das dietas de restrição de calorias, e concluíram que essas dietas são um preditor consistente de ganho de peso. Eles observaram que até dois terços das pessoas recuperaram mais peso do que haviam perdido.

Uma vez que a nutrição não é uma ciência exata, alguns fatores devem ser levados em consideração ao induzir a perda de peso, os quais estão além do conceito de termodinâmica, como adaptações bioquímicas, hormonais, fisiológicas, psicológicas, emocionais, genéticas e epigenéticas, respostas individuais para treinamento, fatores religiosos e sociais que se influenciam mutuamente (Figura 1) (Matarese; Pories, 2014).

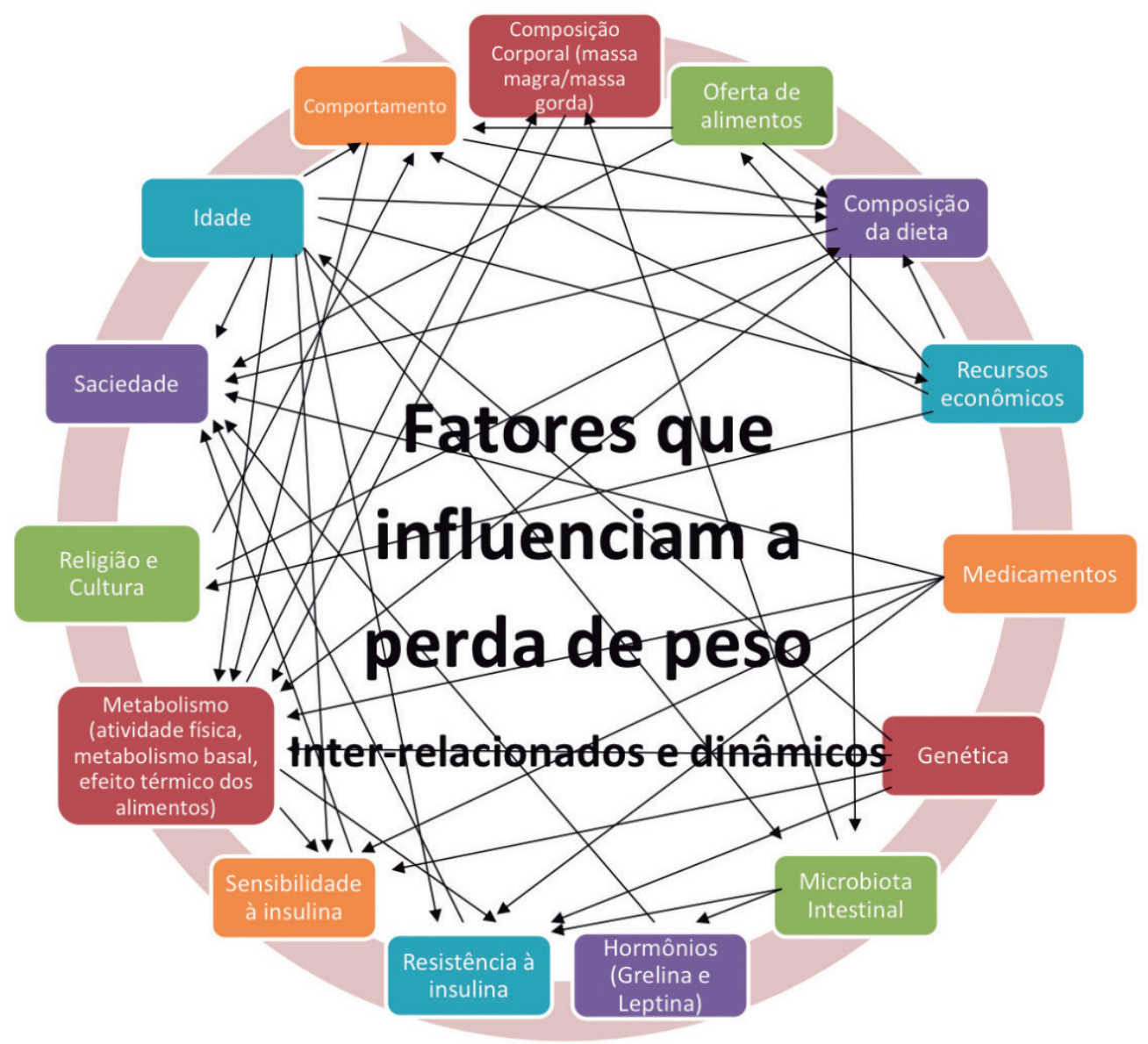

Fonte: Adaptado de Matarese e Pories (2014).

Figura 1 - Fatores que influenciam a perda de peso.

Embora as diretrizes de tratamento para obesidade recomendem mudança de comportamento, pouca ênfase se dá à criação de novos hábitos. O foco prin- 
cipal segue no cálculo de calorias e macronutrientes e na busca pela combinação que melhor promova perda de peso, num contexto no qual, mesmo tendo respaldo científico, a simples quantidade de dietas existentes já mostra que nenhuma tem grande sucesso. Para haver perda de peso sustentável e duradoura são necessários adoção e incorporação de novas rotinas (Dayan et al., 2018).

Todos esses achados corroboram as evidências de que as atuais estratégias de tratamento da obesidade não conseguem sustentar a perda de peso, abrindo espaço para novas técnicas, não baseadas na prescrição dietética, mas principalmente na criação de uma nova mentalidade para saúde, bem-estar e consumo alimentar, como o processo de Coaching Nutricional, que surge como uma forma inovadora de trabalho em saúde e nutrição. O Coaching Nutricional é um processo de parceria entre o coach e o coachee, no qual o profissional apoia o cliente e o ajuda a atingir seus próprios objetivos de melhoria do bem-estar. Geralmente, ocorre facilitando o processo de aprendizagem, e é focado em metas e soluções, sempre olhando para o futuro e não para o passado, como a psicoterapia (Lancha Jr. et al., 2018). A abordagem de Coaching Nutricional se origina do Coahing de Bem-Estar e Saúde.

Conforme proposto pelo Consórcio Internacional de Coaches de Saúde e Bem-Estar (2017),

[...] coaching é um processo centrado no cliente para facilitar e capacitar o cliente a atingir metas autodeterminadas relacionadas à saúde e ao bem-estar. O coaching de sucesso ocorre quando os coaches aplicam conhecimentos e habilidades claramente definidos para que os clientes mobilizem fortalezas internas e recursos externos para uma mudança sustentável.

Como mencionado antes, todos os objetivos são autodeterminados pelo cliente e alinhados com seus valores, honrando que cada um é um especialista em sua própria vida. Um processo de coaching começa com um profissional com atitudes respeitosas e sem julgamento, que acredita que o cliente (não paciente, uma vez que esteja ativo no processo) tem todas as respostas que precisa. Ele guia o coachee e o apoia em fazer planos para ação, dando-lhe confiança e capacitando-o a dirigir sua própria vida. Combinando teoria de psicologia positiva, entrevista motivacional, modelo transteórico de mudança de comportamento, perguntas abertas, escuta ativa, comunicação não violenta e ferramentas para mudança de comportamento, o processo encoraja o cliente a superar a resistência a mudança e transformar objetivos teóricos em ações práticas, passo a passo (Wolever et al., 2013; Lancha Jr. et al., 2018). Nesse contexto, o Coaching Nutricional surgiu como uma estratégia comportamental que aplica técnicas de coaching de saúde e bem-estar para promover mudanças sustentáveis nos hábitos alimentares (Lancha Jr. et al., 2018).

É possível tratar o diabetes tipo 2 simplesmente alterando a dieta e aumentando o exercício, nesse caso os médicos não vão apenas dar um hipoglicemiante oral para tratar a doença, mas realmente melhorar a forma como os pacientes 
podem cuidar de si e a forma como eles podem influenciar a sua própria saúde (O'Hara et al., 2014). Esse conceito baseia-se na literatura do "coaching", que na relação entre um "coach" (profissional de saúde) e um "coachee" (cliente), é o "coachee" que conduz seu próprio processo de mudança. Quando profissionais de saúde praticam e vivem através da medicina de estilo de vida eles transferem a responsabilidade do profissional de saúde ao paciente. O profissional não vai dizer ao cliente o que fazer, mas sim buscarão juntos uma alternativa, na qual o profissional de saúde irá negociar a prescrição com seu paciente. Uma importante citação da Margareth Moore, da Wellcoaches, explica claramente essa mudança: "a forma como os profissionais de saúde geralmente se comportam é como se lutassem contra seus pacientes, mas quando entram no relacionamento de coaching e estilo de vida, eles dançam com seus pacientes".

O profissional de saúde treinado em abordagens comportamentais e em competências de coaching tem mais recursos para gerenciar seu próprio autocuidado. Essa é uma preocupação crescente na área tendo em vista que alguns estudos indicam que um em cada três médicos apresenta sintomas de burnout (Shanafelt, 2009). O burnout é uma síndrome caracterizada pela perda de entusiasmo pelo trabalho (exaustão emocional), pelo tratamento de pessoas como se fossem objetos (despersonalização) e uma percepção de que o trabalho não é mais significativo (baixa realização profissional). As consequências da despersonalização são o distanciamento médico-paciente, não possibilitando um atendimento humanizado e centrado nas necessidades do paciente (Moreira et al., 2018). A formação que prepara os diversos profissionais da área de saúde para trabalhar a partir de escuta ativa, presença e não julgamento é um recurso importante na agenda da humanização do sistema, e como consequência tem impacto importante nos desfechos clínicos.

O processo de coaching também provou reduzir os custos nos cuidados de saúde. Um estudo que aplicou técnicas de Coaching de Saúde e Bem-Estar em 512 indivíduos com Doença Cardiovascular (DCV), combinado com 512 controles, encontrou uma redução de US\$ 19.419 por pessoa naqueles que receberam quatro ou mais sessões no intervalo de seis meses (Byrnes et al., 2017). Essas técnicas também podem ser aplicadas usando telefones celulares. Pearson et al. (2013), em uma intervenção telefônica de 12 semanas, avaliaram e compararam a eficácia de duas abordagens diferentes para perda de peso e prevenção de diabetes tipo 2 em estudantes universitários obesos: um grupo de Coaching (12 semanas em sessões individuais, usando técnicas de entrevista motivacional, perguntas abertas, reais motivações intrínsecas para mudança e metas pessoais) versus uma Abordagem de Modificação Comportamental para perda de peso (programa LEARN - um programa que torna a perda de peso uma meta explícita e se concentra mais nos níveis de ingestão de alimentos com base em prescrições externas e restrição calórica), que também recebia sessões semanais, mas sem o uso de técnicas de coaching. Ao final da intervenção, apesar de o segundo 
grupo ter maior perda de peso, o primeiro diminuiu mais a ingestão calórica do que o grupo LEARN, apresentando melhores hábitos alimentares.

Existe um consenso emergente entre os formuladores de políticas públicas de saúde, organizações profissionais, clínicos e pacientes, em muitas nações, de que os cuidados de saúde exigem mudanças substanciais. Grande parte do dinheiro é direcionada para o tratamento de doenças associadas ao estilo de vida pouco saudável e poderia ser poupada se hábitos de vida mais saudáveis fossem estimulados da maneira correta. Já outros entendem o potencial do processo de coaching com relação aos serviços de apoio à alimentação saudável, atividade física, controle de peso e de doenças crônicas, e esperam usar o serviço de coaching sustentado na promoção do uso ou participação em serviços de prevenção/bem-estar. Além disso, o aconselhamento médico tradicional para "perder peso" muitas vezes resulta em mais risco pela probabilidade do ciclo perda-reganho de peso.

Há relatos afirmando que os profissionais de saúde muitas vezes não possuem conhecimento e habilidade para aconselhar um paciente sobre intervenções no estilo de vida. Esses profissionais aprendem e acreditam que as pessoas mudam pelos fatos, fear (medo) e força ( 3 Fs theory). Normalmente listam todas as consequências para a saúde de estar com sobrepeso, e todas as estatísticas de risco. Gostam de acreditar que as pessoas são racionais, que informação gera mudança, que conhecimento é poder e que o medo muda. Mas será que as pessoas não sabem que excesso de gordura corporal faz mal à saúde? Será que as pessoas não sabem que fumar aumenta risco de câncer? Não sabem que ser sedentário reduz a expectativa de vida? A resposta parece bastante óbvia. No livro Mude ou morra, de Alan Deutschman (2007), o autor traz vários relatos de estudos que mostram que mesmo após um grave problema de saúde, como um infarto, as pessoas mudam por pouco tempo. Cerca de dois anos após o evento, entre 80\%-90\% dos pacientes voltaram aos hábitos antigos, não saudáveis. O autor então coloca que nem o medo de morrer é capaz de promover mudanças duradouras e significativas.

A chave para mudança estaria, portanto, em um processo conhecido como 3 Rs: relação, repetição e reestruturação (Deutschman, 2007).

Relação: ter um novo relacionamento emocional com alguém, ou algum grupo que traga de volta a esperança de que a mudança é possível. Voltar a acreditar que há outra solução para os problemas. Criar rapport, vínculo, é o primeiro e mais importante passo na construção desse novo caminho.

Repetição: essa nova relação deve ajudar o cliente a aprender, praticar e adquirir ferramentas e habilidades para a mudança.

Reestruturação: essa nova relação deve ajudar o cliente a aprender novas formas de pensar, a promover mindset. Reestruturar a forma como ele se relaciona e como ele vê a vida, a alimentação, controle de estresse e etc.

Existe uma frase que diz: "o cliente não se importa com o quanto você sabe, até que ele saiba o quanto você se importa" (Pereira-Lancha, 2018). 
A estratégia de coaching de bem-estar e saúde fornece técnicas e ferramentas ao profissional de saúde destinadas a capacitar um paciente (cliente) a fazer mudanças no estilo de vida saudável, trazendo um novo potencial para o sucesso do gerenciamento de peso. O processo de coaching apoia o desenvolvimento de um relacionamento de ajuda, incentivando o paciente a identificar sua visão, necessidades e objetivos. Além disso, o coaching visa ajudar na organização de rotinas e prioridades, enquanto coloca o paciente no controle de seu destino de saúde.

Com essa visão, no segundo semestre de 2018 foi oferecida pela primeira vez na Universidade de São Paulo a disciplina em Coaching de Bem-Estar e Saúde, na Escola de Educação Física e Esporte (EEFE-USP), optativa para estudantes da graduação da USP de cursos na área da saúde. Também está em desenvolvimento, na EEFE-USP, um trabalho de pós-graduação que aplica técnicas de Coaching de Bem-Estar e Saúde em indivíduos com sobrepeso ou obesidade, combinados com um programa de atividade física, para avaliar seu impacto na composição corporal, qualidade de vida, prática de atividade física e ingestão alimentar de mulheres. Até o presente momento 13 sujeitos completaram 12 sessões de Coaching de Bem-Estar e Saúde. Após esse período, observou-se perda de peso corporal $(-2,6 \mathrm{~kg})$ e massa gorda $(-1,91 \mathrm{~kg})$, mas pouca perda de massa magra $(-0,25 \mathrm{~kg})$, sugerindo eficiência da metodologia e do programa em promover melhora de composição corporal, uma vez que praticamente todo o peso perdido foi de massa gordurosa. Ainda, observou-se melhora em todos os aspectos de Qualidade de Vida autoavaliados, ou seja, nos domínios físico, psicológico, nas relações sociais, nas relações com o meio ambiente e na qualidade de vida geral avaliada. Tudo isso evidencia um reconhecimento sobre uma tendência e a necessidade de preparação do profissional. Mudanças de comportamento relacionadas à saúde têm enorme potencial em reduzir mortalidade, morbidade e custos dos cuidados de saúde, o que fornece ampla motivação para o conceito de medicina de estilo de vida (Pereira-Lancha; Lancha Jr., 2017).

\section{Referências}

BYRNES, J. et al. Coaching Patients Saves Lives and Money. Am J Med, Amsterdan, v.131, n.4, p.415- 21, 2017.

CONSÓRCIO INTERNACIONAL DE COACHES DE SAÚDE E BEM-ESTAR 2017, https://ichwc.org/wp-content/uploads/2015/03/ICHWCHealthWellnessCoachScopeofPractice-FinalFeb12017.pdf

DAYAN, P. H. et al. A New Clinical Perspective: Treating Obesity with Nutritional Coaching v. Energy-restricted Diets. Nutrition, Amsterdan, 2018, in press.

DEUTSCHMAN, A. Mude ou morra. São Paulo: Best Seller, 2007.

FIELDS, A. E. et al. Relation between dieting and weight change among preadolescents and adolescents. Pediatrics, Illinois, v.112, p.900-6, 2003. 
INSTITUTO COALIZÃO SAÚDE. Coalizão Saúde Brasil: uma agenda para transformar o sistema de saúde (2017). Disponível em: <http://icos.org.br/wp-content/ uploads/2017/04/livro_projeto.pdf>. Acesso em: 1 set. 2018.

LANCHA JUNIOR, A. H.; SFORZO, G. A.; PEREIRA-LANCHA, L. O. Improving nutritional habits with no diet prescription: details of a nutritional coaching process. American Journal of Lifestyle Medicine, California, v.12, n.2, p.160-5, 2018.

MANN, T. et al. Medicare's search for effective obesity treatments: diets are not the answer. Am Psychol, Whashington, v.62, p.220-33, 2007.

MATARESE, L. E.; PORIES, W. J. Adult Weight Loss Diets: Metabolic Effects and Outcomes. Nutrition in Clinical Practice, California, v.29, n.6, p.759-67, 2014.

MOKDAD, A. H. et al. Actual causes of death in the United States, 2000. JAMA, Chicago, v.291, n.10, p.1238-45, 2004.

MOREIRA, H. A.; SOUZA, K. N.; YAMAGUCHI, M. U. Síndrome de Brunout em médicos: uma revisão sistemática. Rev Bras Saúde Ocup, São Paulo, v.43, n.3, pl-11, 2018.

NG, M. et al. Global, regional, and national prevalence of overweight and obesity in children and adults during 1980-2013: a systematic analysis for the Global Burden of Disease Study 2013. The Lancet, New York, v.384, n.9945, p.766-81, 2014.

O'HARA, B. J. et al. Longer Term Impact of the Mass Media Campaign to Promote the GetHealthy Information and Coaching Service®: Increasing the Saliency of a New Public Health Program. Health Promot Pract, California, v.15, p.828-38, 2014.

PEARSON, E. S. et al. The CHANGE Program: Comparing an interactive vs. prescriptive approach to self-management among university students with obesity. Can J Diabetes, Amsterdã, v.37, n.1, p.4-11, 2013.

PEREIRA-LANCHA, L. O. P. Estratégias educativas, coaching e comportamento. In: LARA, R. S.; NATACCI, L. Nutrição: visão atual e do futuro. Barueri: Manole, 2018. p.101-9.

PEREIRA-LANCHA, L. O.; LANCHA JUNIOR, A. H. Manual de coaching de bem estar e saúde. Barueri: Manole, 2017. p.130.

SHANAFELT, T. D. Enhancing Meaning in Work - A prescription for preventing physician burnout and promotiong patient-centered care. JAMA, Chicago, v.302, n.12, p.1338-40, 2009.

WOLEVER, R. Q. et al. A Systematic Review of the Literature on Health and Wellness Coaching: Defining a Key Behavioral intervention in Healthcare. Global Advances in Health and Medicine, California, v.2, n.4, p.38-57, 2013.

RESUMO - De acordo com o último relatório da Vigitel 2017 as Doenças Crônicas Não Transmissíveis (DCNT) são responsáveis por $68 \%$ de um total de 38 milhões de mortes ocorridas no mundo em 2012. No Brasil, esse cenário não é diferente: em 2011 as DCNT foram responsáveis por $68,3 \%$ do total de mortes. Milhares de dólares são gastos hoje com políticas que estimulam hábitos de vida mais saudável, mas essa estratégia, por si só, tem continuamente falhado em produzir resultados consistentes e duradouros. É necessário uma nova abordagem, que promova mudanças no estilo de vida, possibili- 
tando a melhora dos parâmetros de saúde das pessoas. O processo de coaching apoia o desenvolvimento de um relacionamento de ajuda, incentivando o paciente a identificar sua visão, necessidades e objetivos. Além disso, o coaching visa ajudar na organização de rotinas e prioridades, enquanto coloca o paciente no controle de seu destino de saúde.

PALAVRAS-CHAVE: Coaching de saúde, Estilo de vida, Doenças crônicas.

ABSTRACT - According to the latest Vigitel 2017 report, non-communicable chronic diseases accounted for $68 \%$ of a total of 38 million deaths worldwide in 2012. In Brazil, the scenario is no different; in 2011 the non-communicable diseases were responsible for $68.3 \%$ of all deaths. Thousands of dollars are spent today on policies that encourage healthier living habits, but this strategy, by itself, has continually failed to produce consistent and long-lasting results. A new approach is needed that promotes lifestyle changes, making it possible to improve people's health parameters. The process of coaching supports the development of a help-oriented relationship, encouraging patients to identify their vision, needs and goals. In addition, coaching aims to assist in the organization of routines and priorities, while putting patients in control of their health end-goal.

KEYWORDS: Health coaching, Lifestyle, Chronic diseases.

Luciana Oquendo Pereira-Lancha é bacharel em Esporte, nutricionista, doutora em Ciências pelo Instituto de Ciências Biomédicas da USP, coach em saúde e bem-estar Wellcoaches School of Coaches (ACSM), diretora do Instituto de Bem-Estar e Saúde (Ibes). @- luciana.lancha@gmail.com / https://orcid.org/0000-0001-6290-0336

Daniele Kallas é mestre em Ciências da Saúde pela Universidade Federal de São Paulo (Unifesp), coach em saúde e bem-estar - Wellcoaches School of Coaches, supervisora em Coaching pela CSA Londres. @ - daniele@coachdesaude.com.br https://orcid.org/0000-0002-9417-8429

Paula Helena Dayan é nutricionista, mestranda pela Escola de Educação Física e Esporte da Universidade de São Paulo (USP). @ - nutri.pauladayan@gmail.com https://orcid.org/0000-0002-5510-7898

Antonio Herbert Lancha Junior é professor titular de Nutrição da Escola de Educação Física e Esporte da Universidade de São Paulo (USP) e coach em saúde e bem-estar Wellcoaches School of Coaches (ACSM). @ - lanchajr@usp.br

https://orcid.org/0000-0003-2128-1182

Recebido em 12.12.2018 e aceito em 5.2.2018.

I Instituto de Bem-Estar e Saúde, São Paulo, São Paulo, Brasil.

II Universidade Federal de São Paulo, São Paulo, São Paulo, Brasil.

III e IV Escola de Educação Física e Esporte, Universidade de São Paulo, São Paulo, São Paulo, Brasil. 